\title{
FAÇADE ET REVERS D'UNE ARCHITECTURE MUSICALE SOLENNELLE \\ LA STRUCTURE DE L'EPICEDION HARMONICUM DE JACOBUS HANDL (GALLUS) ET SES ÉNIGMES NON RÉSOLUES*
}

\author{
MARC DESMET \\ Université Jean Monnet, Saint-Etienne
}

Izvleček: Ob smrti zabrdoviškega opata $C$. Schönauerja je J. Handl leta 1589 izdal Epicedion Harmonicum. To delo, ki se je doslej obravnavalo kot ena trodelna kompozicija, sestoji $v$ resnici iz treh samostojnih kompozicij, objavljenih $v$ enem zvezku. Na njihovo samostojnost kažejo tako njihova besedila, glasbene lastnosti kot tudi zgodovinske okoliščine njihovega nastanka.

Ključne besede: Jacobus Handl - Gallus, Caspar Schönauer, epicedium

\begin{abstract}
Upon the death of Zabrdovice Abbot Caspar Schönauer, Jakob Handl published his Epicedion Harmonicum in 1589. This work, which has heretofore been viewed as a single three-part composition, consists actually of three independent compositions published in one volume. Their independence is indicated by their texts, musical characteristics, and the historical circumstances surrounding their creation.
\end{abstract}

Keywords: Jacobus Handl - Gallus, Caspar Schönauer, epicedium

L'abondante bibliographie consacrée à Jacobus Handl ${ }^{1}$ ne semble pas avoir réservé jusqu'ici de commentaires autres que circonstanciels à l'Epicedion Harmonicum. ${ }^{2}$ Le fait s'explique aisément lorsque l'on sait que cette publication, datée de 1589 , n'a survécu qu'en un unique exemplaire incomplet, ${ }^{3}$ ne permettant guère de jugement avancé sur son contenu musical. À l'occasion de l'édition critique du recueil entreprise dans le cadre des Monumenta Artis

* Institut d'Histoire de la Pensée Classique CNRS UMR 5037. - Mes chaleureux remerciements vont à M.me Janine Hache pour son aide dans la correcte compréhension des textes latins cités aux pages 105, 111-112 et 114, ainsi qu'à M. Vladimír Maňas (Université de Brno) pour son généreux secours documentaire.

1 On se reportera naturellement à la monographie la plus récente et la plus complète sur le compositeur, celle de Marko Motnik, Jacob Handl-Gallus: Werk-Überlieferung-Rezeption, dont la bibliographie couvre 50 pages très denses.

2 Voir en particulier Mantuani, « Einleitung », XX, XXVII, XXVIII ; Straková, « Jakob HandlGallus und die Böhmischen Länder », 13-14; Cvetko, Iacobus Hándl Gallus vocatus Carniolanus, $42-46$.

3 Originellement conservé à l'Institut de Musique Religieuse de Wrocław, l'ouvrage fait aujourd'hui partie des collections de la Bibliothèque Universitaire de Varsovie. 
Musicae Sloveniae,${ }^{4}$ il apparaît à présent possible de détailler ce qui constitue la substance parfois inattendue de ce cas isolé au sein de la galaxie Gallus.

L'ouvrage se présente comme un « tombeau » musical, une déploration composée par Handl à la mémoire de Caspar Schönauer, abbé du monastère prémontré de Zábrdovice (all. : Obrowitz, parfois Zabrdowitz) ${ }^{5}$ à proximité de Brno, ${ }^{6}$ et dont le décès était survenu le $1^{\text {er }}$ janvier de l'année 1589. Ce personnage important gouvernait l'abbaye depuis 1568 et avait également sous sa juridiction les abbayes de Želiv (lat. : Siloë ; all. : Seelau) en Bohême et de Nová Říše (all. : Neureisch ou Neu-Reusch) près de Jihlava (all. : Iglau) en Moravie. Il n'est pas exagéré de voir en lui le protecteur ayant le plus marqué la carrière du musicien Gallus. Non seulement on retrouve son nom d'un bout à l'autre du parcours biographique du musicien, mais les termes mêmes qu'emploie Handl à son égard témoignent d'une familiarité et d'une reconnaissance vraie qui tranchent bien souvent sur les politesses de commande propres aux textes de dédicace. Le fait que l'Epicedion soit lui-même assorti d'une longue préface de Gallus, qui constitue le texte le plus original et le plus important qui nous soit parvenu de la main du compositeur, est une autre preuve indirecte de l'importance de ce personnage pour le musicien. Texte de préface et composition musicale occupent d'ailleurs une proportion à peu près égale dans la publication, ce qui fait de cet Epicedion une exception aussi bien dans les publications de Gallus que dans les éditions de Jiří Nigrin (Georgius Nigrinus), l’imprimeur attitré du musicien à Prague, et dont les autres Epicedia, ${ }^{7}$ tous littéraires, ne présentent pas les mêmes caractéristiques. Avec son titre imprimé de façon très solennelle, son texte d'introduction aussi important que la musique elle-même et sa musique enfin, composée majoritairement dans l'effectif imposant de 8 voix réparties en deux chœurs, l'Epicedion Harmonicum se présente sous l'allure d'une architecture impeccable dont une lecture de détail révèle pourtant quelques zones d'ombre. C'est à l'examen de ce contraste que sont consacrées les lignes qui suivent.

4 Iacobus Handl-Gallus, Undique flammatis; Epicedion harmonicum, ed. Klemen Grabnar, critical ed. Marko Motnik and Marc Desmet, Monumenta Artis Musicae Sloveniae, Supplementa 4.

5 Nous donnons les toponymies alternatives dans les seuls cas où celles-ci apparaissent dans les sources originales citées dans cet article, partout ailleurs nous utilisons par convention la toponymie actuellement valide.

6 Zábrdovice fait aujourd'hui partie de la ville de Brno. Il ne reste rien du monastère originel, détruit à l'époque de Joseph II pour être transformé en hôpital militaire. L'église de l'Assomption de la Vierge encore visible aujourd'hui, se tenait à proximité du monastère. Sa construction échelonnée de 1661 à 1668 sous la direction de l'architecte Giovanni Pietro Tencalla est d'un style baroque bien différent des bâtiments fréquentés par Jacobus Handl du temps où celui-ci vivait au monastère.

7 Quatre autres publications, littéraires, éditées par Jiří Nigrin portent le titre d'Epicedion : Jiří Teplicenus, Epikédion in obitum [...] Ioannis senioris Wartensis a Warta (1574) ; Jan Kerghelius, Epicedion pientissimae [...] d. Sabinae Fundein [...] coniugis Balthazari Klesel (1578) ; Jan Colessius z Rakanova, Epicedion de obitu [...] mag. Martini Rakowsky de Rakow (1580) ; Vincentius Pistalocius, Epicedion in fatalem obitum [...] d. Mariae baron. de Molarth [...] coniugis d. Christophori iunioris Popelii b. de Lobkovitio [...] carmine elegiaco conscriptum (1580). Ces éditions n'épuisent pas le total des publications éditées à l'occasion de funérailles, lesquelles pouvaient également se présenter sous d'autres titres, Epitaphia, Carmen in obitum etc. Voir la liste des éditions Nigrin dressée par Mirjam Bohatcová et Josef Hejnic dans « Knihtiskař Jiř́ík Nigrin a jednolistové « Prorotcví » Jindřicha Demetriana », 102-123. 


\section{Le dédicataire et « Gallus » : la mutuelle estime d'un abbé artiste et d'un musicien des monastères}

Sur Caspar Schönauer, les commentateurs ont en général insisté sur le rôle d'infatigable bâtisseur et de rénovateur des institutions monastiques dont il eut la charge. ${ }^{8}$ Schönauer héritait en effet de fondations dont les bâtiments délabrés, ayant été fort endommagés par les guerres hussites ou les incursions turques, n'apparaissaient plus guère viables. ${ }^{9}$ Son action de bâtisseur s'est doublée d'un sens artistique souvent souligné dans les sources anciennes..$^{10}$ L'action de Schönauer ne devait pourtant constituer qu'une transition, dans la mesure où les efforts entrepris pour restituer leur dignité et leur decorum à des structures d'origine médiévale ne demeurèrent pas longtemps visibles, les bâtiments restaurés et embellis devant tomber à nouveau au cours de la guerre de Trente Ans, sous le coup des destructions opérées par les armées suédoises en Europe Centrale.

L'historien de l'ordre prémontré Léon Goovaerts ne consacre que quelques lignes à Schönauer, où apparaît pourtant l'éclat littéraire et artistique que lui avait reconnu le poète ecclésiastique tchèque Jiří Bartholdus :11

\section{SCHÖNAUER (Gaspar)}

24e prévôt de Neu-Reisch (1562-1570), ensuite 28e abbé de Zabrdowitz, et par un décret impérial de Maximilien II, également abbé de Siloë. Il rebâtit le couvent de Zabrdowitz. Il mourut le $1^{\mathrm{er}}$ janvier 1589 méritant cet éloge : « de monasterio, re literaria, et Musica meritantissimi ». Ce sont les expressions de Georg. Bartholdus Pontanus von Breitenberg, dans son ouvrage intitulé Panegyrica Jesu Christo [...] sacra. Colon. excud. Gerard Greuenbruch, 1594, 4 , et dédié à Ambroise von Telecz ou Telče, 29e abbé de Zabrdowitz.

La remarque de Goovaerts semble provenir d'une source antérieure, plus détaillée, et qui s'avère probablement la première à faire un lien entre Schönauer et le musicien Handl avant les débuts de la musicologie moderne. Il s'agit de la notice que Jan Bohumír Dlabacz, lui-même moine prémontré de Strahov au tournant des XVIIIe et XIXe siècles, consacre à Schönauer dans son dictionnaire historique des artistes de Bohême et dont la teneur mérite d'être reproduite ici intégralement. ${ }^{12}$ On comprend en effet, à la lire, que si Schönauer méritait d'être inclus dans un dictionnaire à teneur « artistique », c'est précisément en raison du fait qu'il s'était lui-même distingué par des mérites particuliers tant

8 Voir Pospěch, Krátký dějepis bývalého kláštera premonštrátků, 27-28 ; Čermák, Premonstráti v Čechách a na Moravě, 226, 451 et 470.

9 Voir Ardura, Prémontrés, histoire et spiritualité, 167-168; Plouvier, « Pour une visite des abbayes prémontrées de Bohême et de Moravie », 93-96.

${ }^{10}$ Les Sacerrimae Memoriae Inclyti Regni Bohemiae Coronae [...], 6-7, évoquent notamment comment l'église de Zábrdovice dut à Schönauer d'apparaître sous une beauté nouvelle. Ce texte réapparaît dans les Manuscripta historica Bohemiae rassemblés par l'historien Johannes Florianus Hammerschmidt, au tome 8 dans la « Series reverendissimorum dominorum abbatum monasterii Zabrdovicensis seu Obrovicensis in Moravia Ordinis Praemonstratensis prope Brunam », 325v-326r.

${ }^{11}$ Goovaerts, Ecrivains, artistes et savants de l'ordre de Prémontré, vol. 2, 155.

${ }^{12}$ Dlabacz, Allgemeines historisches Künstler-Lexikon für Böhmen und zum Theil auch für Mähren und Schlesien, vol. 3, col. 59-60. 
en ce qui concerne la littérature que la musique, d'après ce que l'on comprend des propos de Pontanus rapportés par Dlabacz :

Schönauer, Caspar, un abbé érudit de deux fondations prémontrées, Obrowitz en Moravie et Seelau en Bohême, décédé le $1^{\text {er }}$ janvier 1589. Georg Barthold Pontanus von Breitenberg loue les mérites en musique de Schönauer dans un message envoyé à Ambros von Telecz, abbé d'Obrowitz en 1595, rendu public dans les Panegyrica Jesu Christo sous la forme des impressions suivantes : "Voici déjà presque six ans qu'après la triste mort de ton excellent prédécesseur, le très révérend Dom Caspar (Schönauer), qui a si bien mérité du monastère en littérature et en musique, il t'a été donné d'accéder à cette dignité, toi qui en étais le plus digne, et tu l'as fait avec une si grande intelligence que j'ai voulu l'honorer par une acclamation métrique improvisée, ornée par une mise en musique de l'excellent musicien Jacob Handelius, pieusement décédé, élevé grâce aux bienfaits du Révérend précédent et aux tiens etc. » G. Georgii Bertholdi Pontani : Panegyrica Jesu Christo - sacra. Coloniae excud. Gerardus Greuenbuch. 1594. in 4. Conservé à la bibliothèque du monastère de Strahov. ${ }^{13}$

Que Handl ait été une personnalité de premier plan dans la vie du monastère de Zábrdovice, et que Schönauer ait grandement contribué à promouvoir le musicien est un fait qui n'apparaît ici qu'en filigrane, mais que l'on peut pleinement percevoir en observant comment le nom de Schönauer réapparaît avec constance au sein des publications du musicien. S'ouvre ici une page de l'histoire de l'abbé, dont seuls les musicologues peuvent avoir l'entière connaissance. Lorsque l'on établit la liste chronologique des mentions de Schönauer dans l'œuvre de Gallus, les occurrences suivantes se présentent, outre l'Epicedion.

1. Selectiores quaedam Missae, Prague 1580 : le volume 3 est dédié à Schönauer par Handl qui salue en lui son « patron le plus honorable » (« patronem suum plurimum honorando »), et souligne combien les « remarquables dispositions envers la musique » de ce dernier le rendent son obligé, pour l'avoir soutenu et accueilli durant ses séjours à l'abbaye.

2. Opus Musicum, Prague 1590 (1591) : le tome IV est dédié au successeur de Schönauer à Zábrdovice, Ambrosius de Telcz, ${ }^{14}$ salué par Handl comme son « très vigilant

13 « Schönauer, Kaspar, ein gelehrter Abt der zwei Prâmonstratenser Stifter Obrowitz in Mähren, und Seelau in Böhmen, der im J. 1589, den 1. Jänner, gestorben ist. Georg Barthold Pontanus von Breitenberg rühmt in der an Ambros von Zelecz, einen Abt von Obrowitz, im Jahre 1595 gerichteten Zuschrift, die er der Panegyrica Jesu Christo vorausgeschickt hat, Schönauers Verdienste um die Musik in folgenden Ausrücken: „Jam sextus propemodum annus volvitur, quo tibi post Reverendi admodum Domini Caspari (Schönauer) praedecessoris tui optimi et de Monasterio, re literaria, et Musica meritissimi, tristem obitum, accessionem hanc dignitatis uti, dignissimo, tam tota mente uti, quam extemporanea metrica acclamatione à Musico optimo Jacobo Handelio pie defuncto, Reverendi Antecessoris, tuisque beneficiis exaltato, numeris musicis expolita gratulatus sum etc.“ G. Georgii Bertholdi Pontani: Panegyrica Jesu Christo - sacra. Coloniae excud. Gerardus Greuenbuch. 1594. in 4. wird in der Stifts Strahöwer Bibliothek aufbewahret. »

${ }^{14}$ Egalement connu comme Ambrosius von Teltsch ou Ambrož Telečský selon le contexte linguistique. Teltsch a pu être identifié par Dragotin Cvetko comme le village de Teleč en Bohême de l'Ouest (Cvetko, Iacobus Hándl Gallus vocatus Carniolanus, 45), mais la ville morave de Telč (all. : Teltsch) constitue également un possibilité, car située près de Jihlava, Brno et Klobouky u Brna, tous centres ayant été liés à l'abbaye de Zábrdovice. 
et très fidèle protecteur ». Handl y rappelle que celui dont il vantait jadis les mérites en sa présence, c'est-à-dire Schönauer, est désormais l'objet de ses louanges par l'entremise de son successeur Ambrosius.

3. Harmoniae Morales, Prague 1589 : le livre 1 commence par une mise en musique d'un extrait de L'Enéide de Virgile, « Dii tibi, si qua pios respectant numina ». La pièce est surmontée à la partie de ténor des initiales « C.A.Z.S. » qui valent pour « Casparo Abbati Zabrdovicensi Siloensique » (à Caspar, Abbé de Zábrdovice et de Želiv). On retrouvera le même cartouche en titre courant de la musique de l'Epicedion.

4. Plus importante encore apparaît la mention de Schönauer dans une préface qui ne lui est pas nommément adressée, celle du premier tome de l'Opus musicum (1586). Cette dédicace qui, de façon très solennelle, chante les louanges des trois évêques de Prague (Martin Medek), Olomouc (Stanislav Pavlovský) et Wrocław (Andreas Jerin), contient l'intéressante remarque suivante :

Bien qu'obtenu sous le coup des demandes réitérées par beaucoup, aucun aiguillon ne fut pourtant plus incisif que celui du R.P. Caspar, l’Abbé de Zábrdovice et de Želiv. Vous connaissez très bien cet homme qui jamais n'eut de cesse d'activer son Coq [Gallus], outre qu'il est lui-même remarquable musicien, protecteur et amateur de la Musique. C'est lui qui m'a conseillé, persuadé et a obtenu que je ne retienne ni ne dissimule plus longtemps cet Opus $[\ldots]^{15}$

On peut imaginer l'importance revêtue par un personnage dont le musicien se permet de rappeler le nom à trois évêques dédicataires, tout en soulignant et d'une façon beaucoup plus précise que dans la formule vague de Pontanus qu'il est lui-même un exceptionnel musicien, mélomane et amateur de musique (« ipse Musicus Musicaeque fautor et amator singularis »). Schönauer musicien ? les propos surprennent quelque peu tant ils paraissent singuliers au regard des maigres informations glanées dans les histoires officielles. Il faut toutefois les considérer comme particulièrement pertinents si l'on veut bien imaginer le jugement et le soutien de l'abbé vis-à-vis du compositeur. Déroulées dans la chronologie, ces quatre mentions dessinent, il faut le souligner, tout l'arc chronologique de la carrière de Gallus. La préface du troisième volume des messes évoque tout d'abord, en 1580, la période plus ancienne, celle où le musicien séjournait à Zábrdovice (probablement dans les années 1574-1575). ${ }^{16}$ La préface du premier tome de l'Opus musicum présente ensuite en 1585 le musicien à trois évêques en plaçant ce dernier sous l'autorité « musicale » de Schönauer. Le premier livre des Harmoniae Morales et le quatrième tome de l'Opus

${ }^{15}$ Voir Jacobus Handl, Opus Musicum, tomus 1, f. 2v : « In conspectu Dei mei, aliquid sensim congestum, $\&$ ferè plus, quam nonum in annum prestum merum afferro, quod etsi multorum assiduæ flagitationes expressunt, nullius tamen calcar acrius fuit, atq ; R.D. Caspari Abbatis Zabrdouicensis ac Syloensis, vobis notissimi viri, qui Gallum suum vrgere nunquam desiit, ipse Musicus, Musicæque fautor et amator singularis. Opus diutius ne premerem, ac sepelirem suasit, persuasit, impetrauit. »

${ }^{16}$ Sur l'établissement d'une chonologie plausible quant aux activités de Gallus dans les années 1570-1580, voir Desmet, « Establishing a chronology of Jacob Handl's printed masses. Evidence and problems", $155-168$. 
Musicum datant des années 1589-1591 sont enfin des hommages à la mémoire du défunt abbé, et des éloges de son successeur.

L'Epicedion Harmonicum vient compléter cette dernière catégorie chronologique, avec un détail qui ne manque pas de surprendre, puisqu'il s'agit de la seule publication de Handl où le surnom « Gallus » apparaît mentionné sur la page de titre. Ce titre apparaît rédigé comme suit :

\begin{abstract}
EPICEDION HARMONICUM / PIAE ET NUNQUAM INTERMORITURAE ME- / MORIAE, CASPARI ABBATIS ZABRDOVICENSIS AC SY- / LOENSIS, VIRI VITAE INTEGRITATE DOCTRINA ET ELO- / quentia praestantissimi, Musarum moecenatis aeque devotissimi ut vigilantissimi, / quiete I. Ianuarij anno ultimae patientiae 89. Vita functi. / ADMODUM REVERENDO / VIRO, DOMINO AMBROSIO TELECZENO, / ABBATI ZABRDOVICENSI, LEGITIMO ILLIUS SUC- / cessori \&c. Domino amico meo instar patroni, cum observantia honorando \&c. / solatii loco offert \& dicat / IACOBUS HANDL, GALLUS DICTUS C. / [Dénomination de la partie] / PRAGAE, EXCUDEBAT GEORGIUS NIGRINUS. / [Filet] / Anno : M.D. LXXXIX.
\end{abstract}

DÉPLORATION MUSICALE, À LA PIEUSE ET JAMAIS PÉRISSABLE MÉMOIRE DE CASPAR, ABBÉ DE ZÁBRDOVICE ET DE ŽELIV, HOMME TRĖS DISTINGUÉ PAR L'INTÉGRITÉ DE SA VIE, SA DOCTRINE ET SON ÉLOQUENCE, très attentionné et affectionné protecteur des Muses, qui quitta sereinement cette vie le $1^{\text {er }}$ janvier de l'an 89 de l'ultime patience. AU TRÈS VÉNÉRABLE SEIGNEUR, DOM AMBROISE DE TELCZ, ABBÉ DE ZÁBRDOVICE SON SUCCESSEUR LÉGITIME, et tout comme lui mon maître, ami et protecteur, et que JACOBUS HANDL, DIT GALLUS C.[arniolus] offre et dédie en son honneur, avec respect, en guise de consolation. Prague, élaboré par Georgius Nigrinus, l'an 1589.

Le fait qu'il s'agisse de l'unique page de titre où l'appellation « Gallus » soit présente peut d'autant paraître étonnant que cette appellation, devenue tellement courante par la suite, s'est progressivement imposée comme un patronyme de substitution dans la musicographie. ${ }^{17} \mathrm{Il}$ faut toutefois rappeler ici que cette appellation n'apparaît que de façon discrète dans les sources élaborées du vivant du compositeur, et ce toujours à la façon d'un sobriquet personnel et non d'un patronyme. Y compris dans le tombeau poétique érigé par les amis du musiciens à son décès, c'est toujours la forme « Iacobus Handl » ou bien « Iacobus Handelius » qui prédomine, laquelle est également la forme retenue également par le frère du compositeur, lequel signe toujours en latin « Georgius Handelius », en allemand « Georg Handl » ou en tchèque « Jiř́́ka Handle ». Il n'est pas inutile de constater à ce sujet que l'appellation « Gallus » n’apparaît que :

${ }^{17}$ Déjà fréquent dans la musicographie du XIXe siècle, et même déjà dans quelques copies musicales anciennes, l'usage de l'appellation « Jacobus Gallus », avec Gallus en substitut du patronyme, est en quelque sorte officialisé par Josip Mantuani dans l'introduction à son édition critique de l'Opus musicum en 1899 (voir Mantuani, « Einleitung », XIII). Mantuani s'en explique en indiquant que l'usage de Gallus vaut à ses yeux pour un pis-aller, l'utilisation du patronyme slovène Petelin n'ayant pu être corroboré par aucune source historique. Ce faisant, Mantuani opère une translation du surnom d'artiste du musicien vers une variante latine de son patronyme dont aucun témoignage de l'époque effectué dans l'entourage de l'auteur ne porte la trace. 
1. En signature de la préface des quatre volumes des messes (1580), le compositeur se désignant comme « Iacobus Handl / Gallus vocatus ».

2. Comme une image poétique utilisée dans les trois poèmes liminaires « À l'auteur» ou « À l'Eglise » (Ad Authorem, Ad Ecclesiam) qui ornent les préfaces des trois premiers tomes de l'Opus musicum lesquels filent l'image du coq, oiseau du jour naissant.

3. Comme une image poétique figurant dans les textes de préface signés par le compositeur :

- « la rude voix du coq » (Missae, volume 4),

- « apporter un soutien au coq / à son coq / à ton coq » (Opus Musicum, tomes I et III),

- « le coq chante / le chant du coq » (Opus Musicum, tomes I et II, Harmoniae Morales).

4. En association au patronyme du compositeur (« Iacobus Handl, Gallus dictus ») dans le fameux portrait gravé qui orne le tombeau poétique ainsi que le tome IV de l'Opus Musicum.

Or c'est précisément cette même formulation « Iacobus Handl, Gallus dictus » que nous trouvons en page de titre de l'Epicedion, seul exemple dans toute l'œuvre, et unique apparition aussi officielle du surnom. Il n'est pas interdit d'y détecter que c'est sans doute au sein des monastères et très certainement auprès de Caspar Schönauer, qui fut son protecteur le plus constant, que cette appellation a probablement dû se trouver la plus utilisée, une hypothèse que semble confirmer le fait que c'est dans les volumes de Messes établis en 1580, alors que Handl achève sa période monastique, ainsi que dans les livres I et II de l'Opus Musicum et dans les Harmoniae Morales, recueils qui peuvent tous, d'une façon ou d'une autre, être mis en relations avec Zábrdovice, que l'appellation apparaît. Ni le frère du compositeur, ni les amis proches qui signent les poèmes du tombeau littéraire édité par Nigrin en 1591, ni même son autre puissant protecteur morave, l'évêque d'Olomouc Stanislav Pavlovský, ne semblent devoir être mis en relation avec l'appellation « Gallus » autant que Caspar Schönauer à Zábrdovice.

\section{La préface et la façade apparente de la publication}

La préface conçue par le musicien au seuil du recueil est la plus longue qui soit connue de lui. Aucune des préfaces pourtant développées de l'Opus Musicum, ni même celle du troisième livre de cette série avec son ample Instructio ad musicos (instruction aux musiciens) ne peut rivaliser avec le flot de rhétorique que constitue le discours introductif de l'Epicedion Harmonicum qui, avec ses plus de 850 mots, ressemble à s'y méprendre à l'hommage solennel prononcé à la mémoire du défunt au moment de la cérémonie de funérailles. Gardons-nous de ne voir cependant dans ce discours que les paroles convenues d'un musicien à son fidèle protecteur. La valeur parfois inattendue de la publication, il faut le souligner, réside dans les éléments concrets que Handl insère dans son hommage, et qui permettent de le considérer comme l'un des principaux biographes de Schönauer. Détaillons ici les éléments les plus marquants de ce discours, rédigé à l'adresse du successeur de Schönauer, et comme en sa présence, Ambroise de Telcz. 


\section{a. Biographie de Schönauer}

Après avoir brièvement introduit son discours en rappelant les bontés de Schönauer à l'égard de ses Muses, et en soulignant que de tous les prélats à l'avoir soutenu, Schönauer fut le plus constant et important dans son aide, Handl en vient à brosser un résumé de la vie de l'abbé Caspar :

Originaire de la ville de Brno, proche de ton monastère, il apprit très tôt la langue morave, principalement à partir des sermons de Thomas Bavorovský, et obtint trois prélatures par la voix unanime du monastère, mais aussi par sa sainte majesté impériale Max. II l'empereur des Romains, la première étant celle de Nová Říše, la fille de votre monastère, puis les deux abbatiats de Želiv et de Zábrdovice, dont tu es, en tant que son légitime successeur, à ton tour maintenant le détenteur. Vous tous qui l'avez connu, me serez témoins du fait qu'il portait toutes ces charges honorifiques avec éclat et ténacité. Et comme s'il s'était agi de ne pas permettre à son mérite de s'endormir, il était reconnu non seulement comme Provincial officiel et percepteur de Brno par l'ordre des états supérieurs de votre margraviat, mais même parfois comme Conseiller privé dans quelques cas plus sérieux. Parmi les prélats, qui d'autre que lui est parvenu d'un esprit aussi pénétrant à écouter simultanément ses interlocuteurs, dicter des textes aux scribes, tout en écrivant lui-même ? Cette faculté lui valait d'être salué par les autres prélats tel un sénateur. Et jours et nuits passés viennent témoigner du fait qu'il ne s'épargnait rien des tâches domestiques. Comme en témoigne la direction de Nová Riše sus-mentionnée, institution fondée et quasiment bâtie par lui. En témoigne aussi le monastère de Zábrdovice sus-mentionné, dans lequel il vécut et mourut, et auquel il consacra des travaux d'édification et de restauration qui en ont fait comme un nouveau LÉON COMTE DE KLOBAUK, son fondateur. Voici en effet ce monastère ; je te prends ici, très digne abbé, ainsi que l'ensemble de tes moines et protégés, à témoin : d'une ruine totale, dont les causes furent multiples, Caspar le fit quasiment revenir, depuis qu'il est restauré, à sa splendeur première. Je ne m'étends pas sur les orgues et autres ornements de piété en l'Eglise, sans oublier non plus les vignes, les étangs, les champs et autres biens du même ordre, qui pour la commodité alimentaire du couvent ont été restaurés, nouvellement disposés ou embellis. À qui revient pourtant la jouissance de tous ces biens, à qui ? à toi, son descendant et successeur favori, au couvent, et aux héritiers futurs de votre bien spirituel. ${ }^{18}$

${ }^{18}$ Voir Jacobus Handl, Epicedion Harmonicum, f. 1v-2r : « Is Brunensi Civitate, Caenobio tuo contigua, oriundus, quam / primum : praecipuè autem ex Postillis Thomae Baworowski : Moravam linguam addisceret, totius Conventus legitimo consensu : imò et Sacra Caesarae Majestatis Maxim : II. Rom : Imp : tres Dictaturas, primo quidem Dictaturam Praepositurae Novoreischensis, Filiolae Caenobii vestri, Deinde Abbatiarum duarum, Syloënsis et Zabrdovicensis, cui tu, ut legitimus succesor praees, alternatim est assequutus. Quas quidem omnes dignitates, quanta cum laude et industria administrabat, vos omnes qui novistis illum, estis mihi testes. Et quum nec illi virtus illius conquiesceret, à superiorum statuum ordine in Marchionatu vestro pro Bernae Provincialis officiali ac exactore, imò et aliquando, pro intimo, in non nullis, magis seriis, Consiliario agnoscebatur. Quis aequè Praelatorum, tam peracri acumine, id ut uno ac eodem tempore, et loquentes audire, scribentibus dictare, ipse etiam scriptitare possit, efficere potuit? Ob quod merito Pater quali Conscriptus à reliquis Praelatis salutabatur. Quam nihil etiam laboribus domesticis pepercerit, perhibent dies ac noctes. Perhibet dicta iam Praepositura Novoreischensis, funditus ab eo ferè exaedificata. Perhibet dictum quoque Coenobium Zabrdovicense, cui in erigendo et restaurando, novus quasi Fundator LEO COMES DE KLOBAUK, et vixit et mortuus est. Id enim Coenobium ille : hic te Presul dignissime, Conventum tuum ac omnes Clientes, pro testimonio appello : ita 
Le portrait sommaire de Schönauer qui émergeait des histoires générales, se précise dans le portrait qu'en fait Handl de plusieurs précisions capitales.

- Le prélat est déclaré avoir appris la " langue morave » ce qui nous renseigne indirectement sur le fait que sa langue maternelle était nécessairement l'allemand, avec laquelle il s'entretenait probablement avec Handl. Le fait que la maîtrise de l'idiome slave ait été acquise par Schönauer grâce à la fréquentation assidue des sermons de Thomas Bavorovský aide par ailleurs à préciser d'une touche subtile l'esprit du personnage. Outre leur perfection linguistique et littéraire en langue tchèque, ces sermons émanaient d'un personnage original, un catholique ayant de nombreuses relations parmi les utraquistes et les luthériens, et dont la teneur de l'inspiration puisait dans l'humanisme érasmisant une dimension iréniste, visant à la concorde entre les confessions. ${ }^{19}$

- Schönauer est également présenté dans son activité concrète de bâtisseur et de rénovateur. Ici s'ensuivent des mentions qui valent d'être soulignées, non seulement architecturales, mais aussi concernant les « vignes, étangs, champs » qui nous rapellent que les fondations monastiques d'Europe centrale généraient une substantielle économie agricole. Les « orgues et autres ornements de piété en l'Eglise » signalent de leur côté la dimension artiste du personnage, sensible à une certaine opulence de l'apparence ornée. La musique de l'Epicedion en donne, avec son chœur à huit voix, elle aussi le témoignage. Zábrdovice émerge du lot des trois institutions dont Schönauer avait la charge. C'est là qu'il " vécut et mourut », et c'est là que les travaux entrepris le mettent sur un pied d'égalité, dans le texte de Handl, avec Léon de Klobauk (Lev z Klobouk), puissant seigneur morave ayant octroyé le terrain de l'abbaye aux prémontrés en 1209.

- Nous voyons enfin apparaitre avec ce portrait de Schönauer un individu affairé aux limites du surmenage, dont « jours et nuits » sont consacrés aux activités domestiques d'intérêt général. L'image de l'abbé capable comme bien peu, nous dit Handl, d'« écouter ses interlocuteurs et de dicter simultanément des textes aux copistes, tout en écrivant lui-même » est saisissante tant elle apparaît concrète à un lecteur d'aujourd'hui, rendant d'autant plus plausible l'hypothèse d'un épuisement prématuré du personnage.

\section{b. Une scène de fiction}

La seconde partie du discours d'hommage tourne le dos à la rétrospective historique pour prendre l'allure d'une mise en scène théâtrale. C'est probablement, de tous les textes qui nous sont parvenus de Handl, le plus littéraire et le plus élaboré dans sa

à ruina extrema, plurimisque nominibus decocta è postliminio quasi ad priscum suum nitorem, reduxit. Sileo in Ecclesia pietatis, Organa et alios ornatu ; extra vero, Vineta, piscinas, agros et id genus alia, pro faciliore Conventus sustentatione, adiuventa, erecta et exculta. Cui autem illa omnia cui ? tibi filio suo successori exoptatissimo, Conventui, et peculii vestri spiritualis, haeredibus futuris. »

${ }^{19}$ Voir Havrlant, « Catholic priest Tomáš Bavorovský $(-1562)$ and the reception of Erasmus in the Bohemian Lands », 235-261. Cette nuance ne devait pas échapper aux tenants d'une contreréforme catholique plus radicale, et il est un fait relevé par les commentateurs que si Schönauer devait apparaître comme le restaurateur zélé de la splendeur première des monastères dont il eut la charge, son souci de la discipline et de la stricte orthodoxie catholique passa au second plan au point qu'une réprimande lui fut adressée à la fin de son abbatiat par l'évêque d'Olomouc Stanislav Pavlovský, voir Pospěch, Krátký dějepis bývalého kláštera premonštrátků, 28. 
construction. La réalité s’y mêle habilement à la fiction pour nous présenter les derniers instants de Schönauer suivis d'un dialogue fictif entre ce dernier et Ambroise de Telcz, sont successeur :

\begin{abstract}
Alors qu'il avait accompli les œuvres sus-dites, il vint à s'affaiblir et fut atteint par les rêts de l'hémiplégie en ce jour où il fit usage des remèdes salutaires à la vie : cette année 89 ayant en effet déjà pleinement commencé, au jour dit de la Circoncision de notre Rédempteur, il se présenta, de toute sa foi, aux rites sacrés, et célébra le sacrifice de la messe à Dieu, ainsi que les Vêpres du même jour, puis rendit son âme au S. Dieu. Alors qu'il voulait donner un ultime salut à ce monde immonde (ainsi que je l'ai entendu de témoins dignes de foi), on le vit murmurer ces paroles : « EN TES MAINS SEIGNEUR JÉSUS CHRIST JE RECOMMANDE MON ESPRIT. Quand tu veux, tu m'apelleras. Me voici tien vivant, et je suis mort, CHRIST, viens plus vite ». Alors qu'il eut proféré pour lui même ces paroles d'une voix profonde, il s'endormit très sereinement en CHRIST, à la façon de ceux que gagne le sommeil. Ainsi notre abbé fut-il reçu depuis cette monstrueuse geôle de mort vers la liberté éternelle. Il me semble l'entendre, très désiré successeur, t'adresser ces paroles : « Mon fils, voici comment je t'ai engendré par génération spirituelle, comment je t'ai introduit en la piété, tout comme l'auraient fait des parents naturels, comment j'ai pu me substituer à des précepteurs, et comment j'ai pu accompagner tes débuts vers les disciplines sacrées lorsque c'était possible, alors que ton âge demandait une plus solide nourriture. C'est toi-même, mon AMBROISE, à qui j'ai confié sous le poids des ans le sceptre de ma succession. Je te prie de ne pas décevoir mon attente. Là où tu déposeras les fondements de la piété, poursuis donc d'un même amour paternel ceux dont j'étais le protecteur et le mécène ; c'est-à-dire ceux qui nous ont servi par un office de plus haut caractère, aussi bien en contribuant à ta propre instruction qu'en embellissant le chœur de notre monastère. Interroge toi-même ton esprit pour deviner quels sont ceux auxquels je fais allusion, et consacre à cela une attention et un soutien efficaces ». Tels sont, très digne abbé, les paroles de ton prédecesseur. ${ }^{20}$
\end{abstract}

${ }^{20}$ Jacobus Handl, Epicedion Harmonicum, f. 2 : « Sed quid multis opus ? ubi ante dicta cum laude perficeret, viribus tandem debilitatus Hemiplexiae telo, die eo, quo animae salutaria adhiberet remedia, est attactus : Anno enim hoc 89. penitus iam decurrente, die Circumcisioni Redemtoris [sic] nostri dicto, praemunivit se suamque fidem sacra synaxi, et sacrificium Missae DEO litavit, atque eodem die eis Vesperam, DN. DEO animam suam reddidit. Is quum ultimum vale huic mundo immundo (idenim à fide dignis accepi) dicere vellet, in haec verba secum mussitare videbatur. IN MANUS TUAS DOMINE JESU CHRISTE SPIRITUM COMMENDO MEUM. Quando voles, hinc me vocabis, En tuus sum vivus, et sum mortuus, CHRISTE veni citius. Haec inquam quum secum profundo pectore versaret, dormiturienti similis, placidissimè in CHRISTO obdormivit. Est igitur, Praesul noster, ex hoc teterrimo mortis carcere in aeternam libertatem ascitus. Hic te exoptatissime successor his verbis alloqui nunc mihi videtur. Mi fili ecce quomodo te de re spirituali generatione progenui, ad pietatem introduxi, instar naturalis parentis, praeceptores substitui, et quum solidiora posceret aetas, quò sacris iniciareris ubi licuit emisi. Te ipsum mi AMBROSI, ingravescentis meae aetatis baculum successionis subordinavi. Quaeso expectationem meam noli eludere. Eos etiam, quorum ego : ubi jaceres pietatis fundamenta : fautor et Mecoenas eram, amore paterno prosequere, qui et te informando, et in Choro Caenobii nostri adornando, praestantiore officii genere, nobis inservierunt. Quos intelligam, tu ipse tuam mentem interroga, ac super eo diligenter attende et intende. Ea sunt, dignissime Praesul antecessoris tui verba. » 
On comprend bien les mérites de ce recours à une hypotypose aussi développée, dans la mesure où il s'agit pour le musicien de s'assurer que les bons égards auxquels Schönauer l'avait accoutumé puissent perdurer en la personne de son successeur. Le dialogue fictif imaginé par le compositeur met donc en scène un Schönauer mourant au moment de confier en personne les rênes du monastère à son successeur Ambroise, en lui demandant en particulier de veiller à celui qui s'est davantage distingué par ses mérites. L'allusion, pour voilée qu'elle soit, n'est pas forcément subtile, d'autant que Handl avait commencé cette préface en rappelant avec assez d'insistance les bontés de Dom Caspar pour ses Muses, et qu'il s'apprête à en faire d'autant dans une conclusion où il recommande justement « ses travaux, lui-même et ses biens » à la générosité continuée d'Ambroise. ${ }^{21}$ Mais notons au passage que Handl se décrit lui-même dans la bouche de Schönauer comme ayant été le précepteur musical d'Ambroise (« en contribuant à ta propre instruction »), et qu'il lui recommande du courage dans la poursuite de l'œuvre de Caspar et ce, au moment où lui présente ce sien « lugubre chant » pour les funérailles de son prédecesseur. Nous voilà donc pleinement instruits d'une proximité réelle du musicien avec la haute hiérarchie du monastère. Ayant contribué lui aussi à l'éclat retrouvé de la fondation, Handl a notamment enseigné la musique au successeur de Caspar et s'apprête à faire résonner son chant de déploration. Ayant franchi la façade, nous sommes à présents prêts à pénétrer dans l'intérieur de l'architecture de l'Epicedion.

\section{L'organisation interne de l'Epicedion : des questions sans réponse et une hypothèse}

Sitôt franchi le seuil de l'architecture apparaît le chant à la mémoire de Caspar, qui commence par les mots : «Ergo jaces claustro ereptus Caspare » (« Ainsi gis-tu, Caspar, enlevé à ton monastère »). Alors qu'il ne fait pas de doute que nous tenons avec cet incipit le début du « carmen lugubre » mentionné par Handl dans la préface, il apparaît pourtant que des questions se posent immédiatement quant aux divisions du texte et à son ordonnancement. Observons tout d'abord le déroulement du texte :

Ergo iaces claustrô ereptus Caspare tuisque Ergo iaces, et te mors violenta rapit.

Heu dolor, Heu fatum infœlix

Claustro, mihi, multis nam cadit ante suum nostra corona diem.

Plangimus ecce melos tibi, te lugemus ademptum

Te pater ô Claustri, maxima cura, tui.
Te voilà donc gisant, Caspar, et à ton monastère soustrait,

Te voilà donc gisant, et c'est toi que la violente mort a ravi.

Hélas douleur, hélas infortuné destin,

Au monastère, à moi-même, à nombre d'entre nous, à tous notre couronne est tombée avant son jour.

Nous lamentons ta perte par la musique que voici, nous te pleurons toi qui a été enlevé.

Toi qui par un soin extrême est le père de ton monastère.

${ }^{21}$ La préface du quatrième tome de l'Opus Musicum (1591), adressée précisément à Ambroise de Telcz, donnera à comprendre que le soutien demandé a bien été octroyé au musicien. 
Legitimo successori gratemur ovantes Ambro- Accueillons par des ovations ton légitime sucsio,

Claustri quê pia cura manet

Sis fœlix opto, multos quoque vivito in annos

ô et præsidium spesque decusque meum.

Prædecessori semper mea carmina curae,

Mens eadem tibi sit, Clare Patrone precor.

Sis fœlix opto, multos quoque vivito in annos,

ô et præsidium spesque decusque meum.

Ambrosi selecte Deo, dignissime præsul, et nunc auspiciis Cæsaris aucte bonis, ingredere ô folix claustrum, rege, vive, faveque te nostri memorem terra polusque beet. cesseur Ambroise,

et que du monastère le soin pieux demeure.

Je souhaite que tu sois heureux, et que tu vives aussi de nombreuses années

ô toi mon protecteur, mon espérance et mon honneur.

Ton prédecesseur était toujours soucieux de mes chants,

et je prie pour que tu les accueille, Brillant Patron, d'un égal agrément.

Je souhaite que tu sois heureux, et que tu vives aussi de nombreuses années

ô toi mon protecteur, mon espérance et mon honneur.

Entre dans ce monastère, ô heureux Ambroise, élu de Dieu,

très digne évêque, et à présent promu par les bonnes grâces de l'Empereur,

sois ici le directeur, sois vivant et bienveillant

et que la terre et le ciel te rendent heureux, dans le souvenir de nous.

On voit immédiatement que seule la première section correspond à la définition d' « Epicedion », c'est-à-dire de chant funèbre. Les deuxième et troisième section sont des vœux pour la prospérité de la direction d'Ambroise de Telcz, et des louanges à l'occasion de son élection. Remarquons par ailleurs que le texte de la première, mais surtout de la seconde section fait apparaître un point de vue personnel. La douleur de la « couronne chue » (« cadit nostra corona ») de la déploration initiale est celle qui se manifeste d'abord « à moi-même » (« mihi »), et dans la seconde partie, c'est l'auteur du texte lui-même qui semble se manifester en évoquant Ambroise comme « mon protecteur, mon espérance et mon honneur » (« et praesidium, spesque decusque meum »), lui rappelant combien le prédecesseur Caspar avait été soucieux de « mes chants » (« mea carmina »). Il semble ainsi faire peu de doute qu'à travers le texte poétique de ces deux parties initiales, les paroles prononcées le soient par le musicien qui salue la mémoire de son ancien protecteur, et forme le vœu d'un soutien continué auprès de la part du nouvel abbé. Ce point de vue personnel s'atténue sensiblement dans la troisième partie pour se réduire à un « dans le souvenir de nous » (« nostri memorem »), au sein d'un texte assez neutre qui remplit, de façon plus brève, le rôle d'une louange, d'un panégyrique d'Ambroise au moment de son accession à la tête du monastère « sous les bons auspices de l'empereur ».

A la lecture de ce texte assez composite dans sa formulation, il semble légitime de s'interroger sur sa conformité avec la page de titre. L'Epicedion Harmonicum annoncé au titre désigne-t-il une vaste composition en trois parties ? Ou bien n'est-il limité, au contraire, qu'à la première section ? On voit que la première possibilité rend assez problématique la présence d'une tonalité de louange sur les deux tiers du contenu littéraire, ce 
qui ne cadre pas avec la définition d'Epicedion. ${ }^{22}$ Le second cas est donc bien davantage adapté, si ce n'est qu'il déséquilibre le contenu du recueil par rapport à son titre. Cette possibilité, la plus plausible, revient donc à considérer que ce recueil, qui porte un titre général adapté à la circonstance du décès de Caspar Schönauer, est en réalité constitué de trois compositions distinctes : une déploration à la mémoire de Caspar d'une part (l'Epicedion proprement dit), et deux louanges adressées à la gloire d'Ambroise, toutes deux distinctes en ce que la première semble énoncée d'un point de vue beaucoup plus personnel, celui du compositeur, alors que la seconde rassemble la matière laudative sur quatre vers assez conventionnels.

Il suffit d'observer plus en détail la composition littéraire et musicale du recueil pour que ce curieux agencement apparaisse sous une lumière quelque peu différente.

D'un point de vue littéraire, il est acquis depuis Mantuani ${ }^{23}$ et dans toute la littérature musicologique récente que l'auteur du texte de l'Epicedion n'est autre que le poète Jiř́i Barthold Pontanus z Braitenberka (1550-1604), le vicaire général de l'évêché de Prague, et dont le nom apparaissait déjà mentionné par Dlabacz en 1815 en rapport avec Caspar Schönauer et Ambroise de Telcz. L'attribution à Pontanus des versets funèbres ou panégyriques de deux prélats aussi importants que deux abbés de Zábrdovice ne présente en effet rien que de très logique si l'on veut bien considérer que, nanti de hautes fonctions ecclésiastiques, ${ }^{24}$ le poète s'était fait comme une spécialité des panégyriques des grands hommes, notamment d'Eglise, et des discours solennels, ainsi que des sermons adaptés à tout le calendrier liturgique. L'association du poète avec Handl évoquée par Pontanus lui-même ne présente d'autre part rien d'étonnant, si l'on veut bien considérer que tous deux étaient des familiers non seulement de l'abbaye de Zábrdovice, mais aussi des évêchés de Prague, Olomouc et Wrocław. On en veut d'ailleurs pour preuve qu'au moins un texte de Pontanus figure dans les Harmoniae Morales mises en musique par Handl, ${ }^{25}$ cette fois dans le registre profane, ce qui confirme au moins des liens cordiaux entre les deux personnages. Mantuani s'appuie, pour établir l'autorité de Pontanus quant au texte de l'Epicedion, sur la même citation que Dlabacz, empruntée à la préface des Panegyrica Jesu Christo d'où la citation est extraite. ${ }^{26} \mathrm{Si}$ l'on observe bien la phrase latine de Pontanus

${ }^{22}$ Epicedion signifie oraison ou poème funèbre.

${ }^{23}$ Mantuani attribue le texte de l'Epicedion à Pontanus dans son ample introduction (« Einleitung») à l'édition critique de l'Opus Musicum. Voir Mantuani, « Einleitung », XVIII.

24 Jiří Bartholdus est un auteur très lié aux évêques de Prague. Il est chanoine de la cathédrale Saint-Guy de Prague et secrétaire de l'évêché, promu en 1593 prévôt du chapitre de la cathédrale puis vicaire général de l'évêché. Son œuvre littéraire, très importante, a été largement diffusée de son temps. Elle comprend, outre de nombreuses compositions poétiques de circonstance, en général liées à des personnalités ecclésiastiques, des recueils de sermons, des hymnes versifiées, des ouvrages historiques et des recueils de panégyriques. Voir Barthold Jiří z Braitenberka, Rukovět' humanistického básnictví v Čechách, vol. 1, 137-167, qui contient le détail de toutes ses publications, avec dépouillement.

25 «Nusquam tuta fides », qui porte le n 11 des Harmoniae Morales (1591) est un texte de Pontanus plus tard publié dans le recueil Mantissa vel appendix, elucubrationum poeticarum Pontanarum, 23.

${ }^{26}$ Il est probable que c'est via Dlabacz que Mantuani a été mis sur la piste du recueil de Pontanus, mais il se peut également que Mantuani ait cité directement Pontanus d'après l'original. 
citée par Dlabacz et par Mantuani, les propos du poète sont pourtant assez neutres et s'adressent à Ambroise uniquement :

Voici déjà presque six ans qu'après la triste mort de ton excellent prédécesseur, le très révérend Dom Caspar (Schönauer), qui a si bien mérité du monastère en littérature et en musique, il t'a été donné d'accéder à cette dignité, toi qui en étais le plus digne, et tu l'as fait avec une si grande intelligence que j'ai voulu l'honorer par une acclamation métrique improvisée, ornée par une mise en musique de l'excellent musicien Jacob Handelius, pieusement décédé, élevé grâce aux bienfaits du Révérend précédent et aux tiens.

Comprenons ces propos : Pontanus s'adresse à Ambroise en lui rappelant que lui, Pontanus, a voulu honorer l'intelligente implication d'Ambroise dans les affaires du monastère en improvisant une acclamation métrique mise en musique par Handl, lequel était justement le protégé de Caspar, puis d'Ambroise. Pontanus ne parle donc absolument pas d'un chant funèbre à la mémoire de Caspar, mais uniquement d'une acclamatio, un terme qui ne peut pas se référer à l'Epicedion de la première partie. ${ }^{27}$ Cela paraît d'autant plus logique que Pontanus s'adresse à Ambroise et que c'est autour de lui seulement que la phrase est construite. Un texte d'acclamation est donc évoqué en toute logique, qui correspond à la célébration de l'accession d'Ambroise à la tête du monastère. Cette citation de Pontanus permet ainsi peut-être d'expliquer au moins en partie la curieuse disposition de la composition, mais elle n'offre aucun indice clair de la paternité du texte. À supposer que le texte auquel Pontanus se réfère dans cette citation doive être mis en relation avec l'Epicedion Harmonicum, alors il est évident qu'il ne peut dans ce cas s'agir de la première partie, funèbre, qui ne répond pas à la dimension d'acclamation. Mais peut-il s'agir de la seconde, où, comme nous venons de le voir, le propos de la première est poursuivi grâce à la continuation de l'expression personnelle (" mes chants, mon protecteur »), lesquels émanent du compositeur ? Cette question doit pour l'instant être réservée afin de la confronter à d'autres indices.

Quatre possibilités semblent donc se présenter que l'on peut résumer par les propositions suivantes :

- Pontanus est l'auteur de tout le texte de l'Epicedion, ce qui revient à dire qu'il a fourni à Handl tout le texte latin versifié dont ce dernier avait besoin, et qu'il s'est en particulier exprimé au nom du musicien dans les deux premières parties, la troisième partie étant une acclamation plus neutre. Inconvénient : aucun indice de cette autorité n'apparaît dans les propos de Pontanus lui-même, qui ne se réfère qu'à une acclamatio en l'honneur de l'intronisation d'Ambroise.

- Pontanus est l'auteur des deux chants de louange en l'honneur d'Ambroise. Inconvénient : dans ses propos, Pontanus ne parle que d'une acclamation improvisée. Autre inconvénient : il paraît difficile d'attribuer à Pontanus la première acclamation sans lui attribuer du même coup l'Epicedion à la mémoire de Caspar, dans la mesure où ces deux

${ }^{27}$ Mantuani, qui avait bien remarqué ce détail, indique pourtant qu'à cet endroit Pontanus passe outre sur le chant funèbre, et n'évoque que l'acclamation. Voir Mantuani, « Einleitung », XVIII, note 1 . 
textes présentent les mêmes caractéristiques, et en particulier l'expression personnelle dans laquelle c'est le compositeur qui s'exprime.

- Pontanus n'est, au sein du recueil Epicedion Harmonicum, l'auteur que d'une courte acclamation métrique célébrant l'accession d'Ambroise de Telcz à la direction de l'abbaye de Zábrdovice. Handl met celle-ci en musique et la fait figurer en conclusion du recueil, à la suite de deux autres compositions plus personnelles sur un texte de son cru, consacrées respectivement à un hommage à la mémoire de Caspar Schönauer ainsi qu'à une louange d'Ambroise de Telcz. Avantage : cette option peut coïncider avec les propos de Pontanus, elle permet d'expliquer également le caractère hétérogène de la publication de Nigrin.

- Pontanus n'est l'auteur d'aucun des textes de l'Epicedion et se réfère dans ses Panegyrica à une acclamation mise en musique par Handl et offerte par le musicien en guise de remerciement, aujourd'hui perdue.

On voit d'emblée que la troisième possibilité présente des avantages absents des autres cas, ce que l'examen de la musique de l'Epicedion vient par ailleurs confirmer. Car la distinction entre les deux premières parties et la dernière devient cette fois criante. S'agit-il d'ailleurs de « parties » d'un ensemble, ou bien de compositions distinctes ? Il semble que sur ce plan aussi, un certain flottement soit perceptible dans les rares et succincts commentaires disponibles dans la littérature secondaire, même si le premier paraît plus fréquent. ${ }^{28}$ Entre le début et la fin de sa carrière, Mantuani semble lui-même avoir successivement qualifié le recueil de composition en trois parties ${ }^{29}$ et d'ouvrage composé de trois chants. ${ }^{30}$ Continuons pour l'instant à qualifier les trois volets de « parties ». Qu'observons-nous?

La première, qui coïncide avec le chant funèbre à la mémoire de Caspar, se présente comme une composition à 8 voix réparties en deux chœurs, de mode ionien par bémol (finale $F$ ). Le ton est solennel, les valeurs rythmiques généralement longues, et l'homophonie prédomine dans une alternance des deux chœurs autours de phrases entières, ou de brèves incises (« Ergo jaces », « Te pater »). À deux reprises, un passage de tutti de dense contrepoint fleuri vient animer la texture différemment, coïncidant avec le cri de douleur (« Heu dolor ! ») d'une part, et à l'expression de la douleur de tout le monastère d'autre part (« Claustro, mihi, multis nam cadit »). Cette première partie fait à mainte reprise entendre une signature musicale typique de Handl, qui est la cadence sur $F$ avec septième degré abaissé (le $E$ bémolisé). Ainsi que souligné plus haut, on note enfin que l'imprimé fait figurer en titre courant les initiales «C.A.Z.S. » à toute cette partie, se référant à Caspar, abbé de Zábrdovice et de Želiv.

La seconde partie, consacrée à l'avènement d'Ambroise, est elle aussi à 8 voix réparties en deux chœurs, et elle aussi en mode ionien sur F. Son allure et sa structure sont néanmoins différentes. On note tout d'abord que les valeurs plus rapides et les rythmes enlevés confèrent à la pièce un tempo plus allant, cependant que les principes d'alternance des deux chœurs apparaissent également plus libres. Les deux premiers vers voient les

${ }^{28}$ Straková, « Jakob Handl-Gallus und die Böhmischen Länder », 13, parle d'une « composition en trois parties » (« eine dreiteilige Komposition »), tout comme Motnik dans sa monographie, Jacob Handl-Gallus: Werk - Überlieferung - Rezeption, 44.

${ }^{29}$ « ... composition musicale en trois parties... » (« glasbotvor v treh delih »), voir Mantuani, « Jakob Gallus », 61.

${ }^{30}$ « ... contient trois chants... ( (« obsega tri speve »), voir Mantuani, « Gallus, Jakob (1550-1591)». 
deux chœurs s'enchaîner de façon irrégulière et légère, avec l'émergence d'un gracieux « Ambrosio » rapidement scandé en alternance. L'impression d'accélération se poursuit avec les vers 3 et 4 ( « Sis foelix ... »), où les deux chœurs finissent par se rejoindre dans une sorte de motus perpetuum lançant et relançant le même motif d'imitation figurant les nombreuses années souhaitées au nouvel abbé. Le vers suivant, qui couronne ce dernier des qualificatifs de « protecteur », d'« espérance » et d'« honneur» revient au sobre rythme déclamé en homophonie de la pièce précédente. C'est ce même rythme déclamatoire qui se poursuit pour évoquer ensuite la figure du prédecesseur Caspar, dans une formule où réapparaît également la cadence typique avec septième degré abaissé rencontrée dans Ergo jaces. La composition se termine par une redite littérale des vers 3 et 4 et de leur animation rythmique et contrapuntique caractéristique. Tout comme les pages imprimées de la première partie avaient été surmontées du cartouche «C.A.Z.S. », nous trouvons ici les initiales «A.A.Z.» en tête des pages imprimées, celle-ci valant pour « Ambrosio Abbati Zabrdovicensi » (à Ambroise, abbé de Zábrdovice).

Avec la dernière partie, la divergence de conception devient, comme nous l'avons annoncé, frappante, quand bien même nous retrouvons le cartouche «A.A.Z. » imprimé en tête des pages. Ces quatre vers d'hommage officiel (et impersonnel) à Ambroise sont mis en musique, mais à quatre voix seulement, et dans le mode dorien par bécarre (finale $D)$. Le ton et l'allure de la composition, beaucoup plus brève que les deux précédentes, évoquent cette fois distinctement les Harmoniae Morales, et présentent en guise de conclusion (à partir de « rege, vive ») une section homophone distincte, à trois temps, d'effet brillant et pulsé.

La différence entre les deux premières parties d'une part, et la troisième partie d'autre part rend immédiatement évident le fait que cette polyphonie à 4 voix n'est pas la conclusion des deux autres. Il ne s'agit donc pas d'une grande composition en plusieurs sections mais d'au moins deux compositions indépendantes. Mais que penser d'autre part des deux polyphonies initiales à 8 voix et toutes deux en $F$ ionien ? Bâtissent-elles la prima et secunda pars d'un unique motet, ou bien faut-il les considérer comme elles aussi indépendantes l'une de l'autre ? La réponse tient sans doute d'une voie médiane entre les deux possibilités. Et si l'on se souvient que c'est la circonstance du décès de Schönauer qui est à l'origine de l'Epicedion, il paraît difficile d'imaginer qu'on ait enchaîné les deux parties à la suite l'une de l'autre, à supposer que l'on ait chanté ces polyphonies au moment des funérailles de Dom Caspar. Mais à considérer que la partie « Caspar » et la partie « Ambroise » sont indépendantes l'une de l'autre, on remarquera que la seconde est faite comme pour se présenter en complète continuité stylistique de la précédente. L'allusion claire adressée au successeur de Caspar sur le fait qu'il prend la suite d'un mécène généreux apparaît ici comme renforcée par les similitudes d'effectif et de modalité des deux compositions, et même par des réminiscences qui, si elles ne concernent que des cadences ou bien l'écriture homophone du centre de la composition, s'avèrent propres à évoquer une parenté de conception avec la première pièce.

L'Epicedion Harmonicum apparaît donc plus clairement à présent sous l'allure d'un recueil de trois compositions distinctes. Il nous faut toutefois revenir sur l'altérité très marquée de la troisième, laquelle forme une bien bancale addition aux deux majestueuses polyphonies principales. Pourquoi cet ajout un peu étrange à l'Epicedion que cette légère 
composition à quatre voix comme complément ? Certes, le sujet est le même, qui renchérit non pas sur la partie « Caspar », mais sur la partie « Ambroise ». Au vu du caractère circonstantiel de la publication, son adjonction n'est pas problématique en soi, mais elle l'est davantage sur le plan de la conception du recueil : un contenu aussi réduit de trois pièces seulement met en effet en évidence une disparité qui ne se remarquerait pas autant dans une anthologie musicale plus vaste. Il paraît difficile d'imaginer que cette composition puisse avoir été conçue avant l'Epicedion, car l'accès d'Ambroise à la tête du monastère n'a pas pu être célébrée musicalement par Handl avant la déploration sur le décès de Caspar. Mais il est tout aussi difficile de la dater de beaucoup après, sachant que l'édition Nigrin date précisément de l'année du décès. Or, le fait même que la troisième partie s'insère finalement assez mal à la suite des deux compositions principales du recueil n'incline pas non plus à penser que Handl aurait conçu cette partie exprès pour le volume. C'est donc probablement à d'autres contraintes ou possibilités qu'il faut attribuer sa présence à la suite des deux autres polyphonies, son inclusion n'ayant été décidée qu'au tout dernier moment.

Parmi ces contraintes, des considérations strictement éditoriales ont sans doute pu jouer leur rôle. Observons en effet que les huit voix des deux pièces précédentes sont réparties en quatre parties séparées, présentant dans chacun des quatre cahiers ${ }^{31}$ le chœur I sur la page de gauche, et le chœur II sur la page de droite. Cantus I et Cantus II sont ainsi en vis-à-vis dans le cahier de Cantus, Altus I et Altus II dans le cahier d'Altus, etc. Cela signifie nécessairement que la fin du deuxième chant coïncide dans tous les cahiers avec une page de droite (fin du chœur II), laissant disponible au verso de cette page un espace vide, c'est-à-dire potentiellement un espace à remplir. La composition Ambrosi selecte Deo occupe précisément cet espace, qui n'utilise donc qu'une seule page sur chacun des quatre cahiers et ne laisse par conséquent à l'imprimeur que la possibilité d'insérer une polyphonie à 4 voix seulement. Le changement d'effectif pourrait s'expliquer par cette contrainte de dernière minute, nécessitant de trouver rapidement une composition à quatre voix permettant de combler un espace autrement laissé vacant.

À son tour, cette contrainte de la rapidité a pu mettre le compositeur dans l'obligation du remploi. Comme nous venons de le voir, la pièce ajoutée est musicalement trop disparate par rapport aux deux compositions principales pour qu'on puisse imaginer qu'elle ait été conçue exprès pour ce recueil. On en déduit donc que puisqu'il s'agissait de remplir un espace vacant, Handl s'est trouvé dans l'obligation de fournir très rapidement à l'imprimeur une pièce déjà prête. Or, comme nous l'avons remarqué le style de cette courte polyphonie à 4 voix est absolument identique à celui des pièces figurant dans les Harmoniae Morales. Comme nous savons par ailleurs que ce dernier recueil est constitué d'un choix de pièces parmi d'autres, ${ }^{32}$ il est parfaitement possible que nous ayons affaire

${ }^{31}$ L'unique exemplaire conservé de l'Epicedion Harmonicum, aujourd'hui à la bibliothèque universitaire de Varsovie, est incomplet du cahier de Tenor, autrement dit des deux voix de Tenor I et Tenor II. Ces deux voix ont été reconstituées dans l'édition critique à paraître du recueil au sein de la série Monumenta Artis Musicae Sloveniae.

${ }^{32}$ Le frère de Gallus, Georg / Jurij Handl, publiera dans les Moralia d'autres pièces du même genre, mais à 5, 6 et 8 voix. Dans la préface, Georg indique que le compositeur avait reçu énormément de textes à mettre en musique, et qu'il aurait poursuivi sa tâche s'il n'était pas prématurément décédé d'épuisement. On sait que toutes les pièces de ce type composées par Gallus n'ont pas 
à une pièce déjà écrite sur ce texte, et fournie in extremis pour combler l'espace vide de la dernière page disponible dans le recueil Epicedion Harmonicum, à moins que Handl ait ici remployé une composition déjà disponible en l'adaptant à un texte de circonstance qui venait de lui être fourni. Notons en effet que la prosodie, en général très soignée chez le compositeur qui fait un large usage de l'homophonie, présente quelques particularités étonnantes dans cet Ambrosi selecte Deo, qui laissent transparaître, si ce n'est de la hâte, du moins une origine possiblement modifiée sur le plan littéraire :

a. L'énoncé de la première phrase à la partie de Cantus accentue curieusement le prénom «Ambrosi » en allongeant la première et la troisième syllabes, c'est-à-dire en laissant de côté la seconde, qui est seule porteuse de l'accent.

b. Le mot « auspiciis » est de même formulé pourvu d'un mélisme curieusement situé sur la troisième syllabe (-ci-) à la partie de Cantus.

c. La répétition triple du fragment syntaxique « polusque beet » à la toute fin de la pièce est assez étrange, alors que la proposition sémantique du passage est conçue comme « terra polusque beet ».

Ces remarques prennent un relief nouveau lorsqu'on les met en parallèle aux conclusions et questions provisoires qui émanaient de la lecture du texte effectuée plus haut. Il s'agissait de déterminer si Pontanus, qui mentionne dans sa lettre à Ambroise être l'auteur d'une acclamation rédigée quasiment sur le champ (« extemporanea ») au moment de son accession à la tête du monastère, pouvait être ou non mis en relation avec l'Epicedion Harmonicum d'une part, et dans quelle proportion d'autre part : tout le texte ? les deux acclamations ? la dernière partie seulement? aucun texte ? Il semble qu'avec la curieuse disposition musicale de la dernière pièce, nous soyons à présent davantage en mesure de formuler une hypothèse plausible. Pontanus faisait état d'une acclamation rédigée sur le champ de façon improvisée. À sa manière, la disposition bancale de la dernière pièce de l'Epicedion Harmonicum trahit elle aussi une espèce de hâte dans la constitution du recueil. Nous pouvons y voir un indice assez sûr qu'il s'agit de l'acclamatio mentionnée par Pontanus. Un nouvel état des connaissances sur le recueil pourra nous servir de provisoire conclusion à cette enquête.

\section{Conclusion : de quoi l'Epicedion Harmonicum est-il constitué ?}

1. Jacobus Handl est l'auteur d'un recueil intitulé Epicedion Harmonicum publié à Prague en 1589 par l'imprimeur libraire Jiří Nigrin (Georgius Nigrinus) lequel a déjà fait paraître de lui quatre volumes de messes en 1580 et trois tomes de motets en 1586 et 1587. Deux autres recueils poursuivront, après ce recueil, la collaboration Nigrin-Handl : les pièces profanes latines intitulées Harmoniae Morales (1590-1591) et le quatrième tome des motets en 1591.

2. Cet Epicedion Harmonicum constitue un hommage à la mémoire d'un protecteur de longue date du compositeur, l'abbé de Zábrdovice Caspar Schönauer. Handl apparaît

été imprimées, un certain nombre d'entre elles ayant été découvertes en manuscrit, qui peuvent lui être attribuées. 
avoir été très lié à ce personnage auquel le liaient un commun intérêt pour la musique et probablement une amitié sincère, depuis une époque où, jeune compositeur, Handl s'exerça à la composition dans les années 1574-1575 dans son monastère.

3. Le recueil contient également deux acclamations célébrant l'accession à la tête du monastère du successeur de Caspar, Ambroise de Telcz, auquel Handl avait assuré une éducation musicale.

4. Il s'agit d'un recueil très personnel, au sein duquel Handl formule dans une longue préface le souhait qu'Ambroise puisse continuer à le soutenir financièrement comme l'avait fait depuis toujours Caspar. Le chant de déploration à la mémoire de Caspar, mais aussi la première acclamation portent cette marque en recourant à des expressions elles aussi personnelles où le compositeur s'exprime en son nom propre, un fait unique au sein de toute son œuvre.

5. De même que le titre et la préface du recueil font une place symétrique au décès de Caspar et à l'intronisation d'Ambroise, la première acclamation à l'adresse d'Ambroise est mise en musique par Handl comme un volet symétrique de la déploration à la mémoire de Caspar. La musique y est à 8 voix en deux chœurs de mode ionien, des tournures similaires s'y remarquent. En revanche, la seconde acclamation, sur un texte beaucoup plus court et plus neutre, est une simple et légère polyphonie à 4 voix conçue en mode dorien. Elle ne forme aucun ensemble cohérent avec les deux autres pièces.

6. Le poète ecclésiastique de l'évêché de Prague, Jiří Barthold Pontanus z Breitenberka, déclare dans une préface adressée à Ambroise de Telcz en 1595, qu'il se souvient s'être impliqué dans les affaires du monastère au moment de l'accession de ce dernier à la tête du monastère. Il se souvient également avoir été remercié de cela en recevant de la part de Handl la mise en musique d'une acclamation versifiée qu'il avait improvisée sur le champ au moment des faits. Ces lignes ont en général été comprises comme signifiant que Pontanus était l'auteur de tout le texte de l'Epicedion Harmonicum.

7. La dernière pièce musicale, très en dehors stylistiquement des deux autres, laisse supposer qu'il s'agit d'un ajout tardif. Des traces d'une adjonction à la hâte sont décelables aussi dans l'adéquation du texte à la musique. Par opposition, la dimension très personnelle de la préface, de la déploration et de la première acclamation invitent à lire les propos de Pontanus en les conservant dans leur sens premier. Le poète évoquant un texte rédigé sur le champ au moment même de l'événement peut avoir été gratifié par Handl d'une mise en musique effectuée elle aussi à la toute dernière minute avant que l'ouvrage ne soit imprimé. Handl aurait alors pu réutiliser les plans d'une polyphonie déjà faite, ou bien composer lui-même très rapidement sur ces quatre vers.

8. L'autorité des deux premiers chants, qui sont la substance principale de l'Epicedion, reste ouverte. Leur dimension très personnelle, et très liée au contenu de la préface peut accréditer l'idée que Handl en soit directement l'auteur. Si l'on doit imaginer que Pontanus en est tout de même l'auteur, alors il faut remarquer que s'exprimant au nom du musicien, il rend à Handl un signalé service permettant à ce dernier d'offrir au monastère de Zábrdovice l'expression artistique de sa situation en des termes particulièrement choisis. ${ }^{33}$

${ }^{33}$ Aucun des textes de Pontanus mentionnés dans le dépouillement complet de son œuvre au sein du Rukovět' humanistického bástnitcví, vol. 1, 137-167, ne semble en tout cas indiquer la présence d'un Epicedion à la mémoire de Caspar Schönauer. 
9. En 1589, Jacobus Handl se trouve à un tournant important de sa carrière et sa situation est fragilisée non seulement par le décès de Caspar Schönauer, mais aussi par le départ de son frère Georgius, qui quitte l'atelier Nigrin pour se rendre probablement à Nuremberg. ${ }^{34}$ Etant à la recherche de nouveaux soutiens, le musicien exprime avec l'Epicedion Harmonicum sa fidélité aux prémontrés de Zábrdovice qui l'ont toujours secondé. Mais ce recueil marque aussi, sans doute pour les mêmes raisons, son rapprochement du milieu de la cour impériale dont Pontanus faisait absolument partie. Son hommage à la mémoire de Caspar Schönauer et à l'élection d'Ambroise de Telcz se double donc en filigrane d'un hommage à Pontanus, qui prend la forme d'une mise en musique à quatre voix de la courte acclamation que le poète déclare lui-même avoir improvisé sur le champ au moment des événements. Ajoutée in extremis à la suite de sa propre composition à huit voix, cette composition confirme les dire du poète, tout en conférant au recueil un déséquilibre interne qui en trahit le caractère circonstancié.

\section{Bibliographie}

Ardura, Bernard. Prémontrés, histoire et spiritualité. Saint-Etienne : Presses de l'Université de Saint-Etienne, 1995.

Barthold Jiří z Braitenberka. Rukovět humanistického básnictví v Čechách. Vol. 1. Ed. Antonín Truhlář et Karel Hrdina. Prague : Nákladem Československé Akademii Věd, 1966.

Bohatcová, Mirjam, et Josef Hejnic. « Knihtiskař Jiřík Nigrin a jednolistové « Prorotcví » Jindřicha Demetriana ». Sborník Narodního Muzea v Praze 35, nº 2 (1981) : 73-134.

Cvetko, Dragotin. Iacobus Hándl Gallus vocatus Carniolanus. Ljubljana : SAZU, 1991.

Čermák, Dominík K. Premonstráti v Čechách a na Moravě. Prague : Cyrillo-Methodějská kněhtiskárna (V. Macan.) - Nákladem kanonie Strahovské, 1877.

Desmet, Marc. « Establishing a chronology of Jacob Handl's printed masses. Evidence and problems ». Dans The Musical heritage of the Jagiellonian era, ed. Paweł Gancarczyk et Agnieszka Leszczyńska, 155-168. Varsovie, Instytut Sztuki PAN, 2012.

___ _ " Typographicum robur fractum: the relationship between Jacob Handl and the printing press ». De Musica Disserenda 3, n 2 (2007) : 11-24.

Dlabacz, Gottried Johann (Jan Bohumír). Allgemeines historisches Künstler-Lexikon für Böhmen und zum Theil auch für Mähren und Schlesien. Vol. 3. Prague : Gottlieb Haase, 1815.

Goovaerts, Léon. Ecrivains, artistes et savants de l'ordre de Prémontré. Dictionnaire bio-bibliographique. 4 vols. Bruxelles : Société Belge de Librairie, 1899-1907. (Réimpr. : Genève : Slatkine, 1971.)

___ Ecrivains, artistes et savants de l'ordre de Prémontré. Dictionnaire bio-bibliographique. Vol. 2. Bruxelles : Société Belge de Librairie, 1903. (Réimpr. : Genève : Slatkine, 1971.)

${ }^{34}$ Voir Desmet, « Typographicum robur fractum, the relationship between Jacob Handl and the printing press », 11-24. 
Hammerschmidt, Johannes Florianus. « Series reverendissimorum dominorum abbatum monasterii Zabrdovicensis seu Obrovicensis in Moravia Ordinis Praemonstratensis prope Brunam ». Dans Manuscripta historica Bohemiae, ed. Johannes Florianus Hammerschmidt, vol. 8, 319r-328v.

Handl, Jacobus. Epicedion Harmonicum. Prague : Jiří Nigrin, 1589.

. Opus Musicum, tomus 1. Prague : Jiří Nigrin, 1586.

Handl-Gallus, Iacobus. Undique flammatis; Epicedion harmonicum. Ed. by Klemen Grabnar. Critical edition by Marko Motnik and Marc Desmet. Monumenta Artis Musicae Sloveniae, Supplementa 4. Ljubljana: Založba ZRC, ZRC SAZU, 2014.

Havrlant, Jaroslav. « Catholic priest Tomáš Bavorovský (-1562) and the reception of Erasmus in the Bohemian Lands ». Dans The Bohemian Reformation and Religious Practice, vol. 8, ed. Zdeněk V. David et David R. Holeton, 235-261. Prague : Filosofiský časopis, Filosofia, 2011.

Kruger, Georg. Sacerrimae Memoriae Inclyti Regni Bohemiae Coronae [...]. Litomyšl : Joannes Arnolt, 1667.

Mantissa vel appendix, elucubrationum poeticarum Pontanarum. Francfort-sur-le-Main : Nicolaus Basseus, 1595.

Mantuani, Josip. « Einleitung ». Dans Jacob Handl (Gallus), Opus Musicum, vol. 1, ed. Emil Bezecný et Josef Mantuani, VII-XXXIII. Vienne : Artaria, 1899.

___. « Jakob Gallus ». Cerkveni glasbenik 14, n 8-9 : 57-65. . « Gallus, Jakob (1550-1591) ». Dans Slovenska biografija. Ljubljana: Slovenska akademija znanosti in umetnosti, Znanstvenoraziskovalni center SAZU (Internet, 14 août 2014). Edition originale : Slovenski bijografski leksikon, vol. 2, ed. Izidor Cankar et al., 202-203. Ljubljana : Zadružna gospodarska banka, 1926.

Motnik, Marko. Jacob Handl-Gallus: Werk-Überlieferung-Rezeption. Tutzing : Hans Schneider, 2012.

Plouvier, Martine. « Pour une visite des abbayes prémontrées de Bohême et de Moravie ».

Dans Actes officiels du $29^{\circ}$ colloque du Centre d'Etudes et de Recherche Prémontrées :

Bohême-Moravie, ed. Martine Plouvier, 79-115. Žd'ár nad Sazavou, 2003.

Pospěch, Josef. Krátký dějepis bývalého kláštera premonštrátkủ, nynějši farnosti a chrámu Páně v Zábrdovicích. Brno : Josef Šnaider, 1869.

Straková, Theodora. « Jakob Handl-Gallus und die Böhmischen Länder ». Muzikološki zbornik 18 (1982) : 5-21.

VIDNO IN PRIKRITO V NEKI VELIČASTNI GLASBENI ARHITEKTURI STRUKTURA EPICEDION HARMONICUM JACOBUSA HANDLA (GALLUSA) IN NJEGOVE NEREŠENE UGANKE

Povzetek

Ob smrti zabrdoviškega opata Casparja Schönauerja je Jacobus Handl - Gallus leta 1589 izdal Epicedion Harmonicum. To delo, ki se ni v celoti ohranilo (manjka tenorski 
zvezek), obsega skladateljev daljši uvod ter samo glasbo. V latinskem uvodu J. Handl oriše življenjsko pot svojega mecena Schönauerja, nato pa se obrne na njegovega naslednika Ambrozija iz Teleča; vzpodbuja ga, naj nadaljuje delo svojega predhodnika, tudi v tem, da ostane mecen tistim, ki jih je podpiral njegov predhodnik.

Sledi sam epicedium. Ta se je v doslejšnji literaturi obravnaval kot ena kompozicija v treh delih, vendar sestoji iz treh samostojnih skladb. Prva je epicedium v pravem pomenu besede, tj. žalostinka za umrlim Schönauerjem; druga je aklamacija novoizvoljenemu opatu Ambroziju kot skladateljevemu novemu mecenu; besedili teh dveh kompozicij sta slogovno podobni in pisani zsta osebnega stališča. Tudi tretja kompozicija je aklamacija novemu opatu, vendar se njeno besedilo slogovno loči od prejšnjih dveh, med drugim tudi v tem, da je neosebno.

Doslej je veljalo, da je vsa tri besedila spesnil Barthold Jiří z Braitenberka (Pontanus), generalni vikar praške škofije. Vedenje o tem izhaja iz Pontanovega zapisa, v katerem pravi, da je v čast novemu opatu Ambroziju priložnostno napisal aklamacijo, ki jo je uglasbil Handl. Iz tega je razvidno, da je Pontanus ob smrti starega in nastopu novega opata napisal le eno besedilo, ki je bilo po svojem tipu aklamacija, ne pa treh besedil, kolikor jih vsebuje Epicedion Harmonicum.

Tri kompozicije Epicedion se razlikujejo tudi glasbeno. Prvi dve sta v jonskem modusu na $F$ in zasnovani sta za dva štiriglasna zbora. Kljub temu ju ni mogoče imeti za eno kompozicijo v dveh delih, saj se nista mogli izvajati skupno: prva se je lahko pela ob Schönauerjevem pogrebu (kjer za drugo gotovo ni bilo mesta), druga pa ob umestitvi novega opata (kjer bi bilo petje prve neprimerno). Tretja kompozicija je napisana za štiriglasni zbor in je v dorskem modusu, kar pomeni, da se glasbeno bistveno loči od prvih dveh.

$\mathrm{V}$ glasovnih zvezkih je tretja kompozicija natisnjena na zadnji strani, ki bi brez nje sicer ostala prazna. To nakazuje, da jo je skladatelj vstavil šele potem, ko se je izkazalo, da je v novem tisku ostalo nekaj prostora, ki bi se ga dalo izkoristiti. Glede na to, da je besedilo te skladbe aklamacija, da se slogovno loči od predhodnih dveh besedil, kar pomeni, da ima drugega avtorja, in da izgleda Handlova uglasbitev narejana na hitro (kot tudi besedilo samo), je mogoče domnevati, da je prav ta kompozicija delo, ki ga v svojem zapisu omenja Pontanus.

\section{THE FACADE AND THE BACK OF A SOLEMN MUSICAL ARCHITECTURE THE STRUCTURE OF EPICEDION HARMONICUM BY JACOBUS GALLUS (JACOB HANDL) AND ITS UNRESOLVED ENIGMAS}

\section{Summary}

Upon the death of Zabrdovice Abbot Caspar Schönauer, Jacobus Handl - Gallus published his Epicedion Harmonicum in 1589. This work, which is not preserved in its entirety (the tenor part is missing), comprises a long introduction by the composer and the music itself. In his Latin introduction Handl describes the life of his patron Schönauer, and then he 
turns to his successor Ambrose from Teleč; he exhorts him to continue the work of his predecessor, including remaining the patron of those that his predecessor had supported.

Next is the epicedium itself. In the literature to date this has been interpreted as one composition in three parts, but it comprises three independent compositions. The first is an epicedium in the true sense of the word: a lamentation for the deceased Schönauer; the second is an acclamation of the newly elected Abbot Ambrose as the composer's new patron. The texts for these two compositions are stylistically similar and written from a personal standpoint. The third composition is also an acclamation of the new abbot, but its text is stylistically distinct from the first two in several ways, including that it is impersonal.

Until now it has been believed that all three texts were written by Georg Barthold Pontanus from Braitenberk, the vicar general of the Prague diocese. This assumption has been based on one of his writings in which he claimed to have written a commemorative acclamation in honor of the new abbot Ambrose, which was then set to music by Handl. This makes it clear that upon the death of the old abbot and appointment of the new one, Pontanus wrote only one text, which was an acclamation type, but not three texts, which is the number contained in the Epicedion Harmonicum.

The three Epicedion compositions also differ musically. The first two are in the Ionian mode on F and are conceived for two four-voice choirs. Despite this, it is impossible to see them as one composition in two parts, because they could not be performed together: the first may have been sung at Schönauer's funeral (which was certainly not the place for the second), and the second upon the installation of the new abbot (where singing the first would have been inappropriate). The third composition is written for a four-voice choir and is in the Dorian mode, which means that musically it is quite distinct from the other two.

In the part books the third composition is printed on the last page, which would have remained blank without it. This indicates that the composer inserted it only when it became clear that the new print would have some leftover space that could be utilized. Considering that the text of this composition is an acclamation and that it is stylistically distinct from the previous two texts, which means that it has a different author, as well as the fact that Handl's musical setting (and the text itself) seems to have been done hastily, it seems likely that this composition is the one Pontanus mentions in his writings. 